\title{
Observación de conductas inseguras en el trabajo: un análisis metodológico*
}

\section{Observation of unsafe behavior at work: A methodological analysis}

Recibido: Marzo 11 de 2010 | Revisado: Mayo 20 de 2010 | Aceptado: Septiembre 21 de 2010

\author{
Orquídea Castilla Ramos** \\ Universidad Simón Bolívar, Miranda, Venezuela
}

SICI: 2011-2777(201203)11:1<311:OCITAM>2.0.CO;2-Q

Para citar este artículo: Castilla, O. (2012). Observación de conductas inseguras en el trabajo: un análisis metodológico. Universitas Psychologica, 11(1), 311-321.

* Artículo teórico.

** Departamento Ciencia y Tecnología del Comportamiento, División de Ciencias Sociales y Humanidades. Edificio de Estudios Generales, Piso 1. Apartado Postal 89000. Valle de Sartenejas. Baruta. Edo. Miranda. Universidad Simón Bolívar. Telfs. 58-212-9063810 / 58-212-9063818 / 58-4142541674.E-mail: ocastilla@usb.ve

\section{RES U MEN}

La observación de conductas inseguras y seguras en el trabajo se emplea en muchas empresas, para la detección de actos inseguros vinculados a accidentes personales o industriales y el diseño estrategias de intervención. El uso de esta técnica en ambientes naturales requiere mucha rigurosidad, tanto en la elaboración de los registros como en su aplicación. En este artículo, se analizan los problemas metodológicos, así como las ventajas y desventajas del uso de esta técnica en ambientes organizacionales, además de las implicaciones para la puesta en práctica de intervenciones psicosociales en una organización. Se concluye que podría emplearse, de manera complementaria, con otras técnicas provenientes de otros enfoques y que su utilidad responde de alguna manera a la cultura de seguridad existente en la organización, cultura que el mismo procedimiento persigue promover, para prevenir y controlar la ocurrencia de futuros accidentes en las empresas. Palabras clave autores:

Conductas seguras, conductas inseguras, percepción de riesgos, prevención, seguridad y salud en el trabajo.

Palabras clave descriptores:

Accidentes laborales, actos inseguros, conducta, observación, prevención, seguridad.

\section{A B S T R A C T}

Observation of unsafe and safe behaviors at work is used in many companies, to known unsafe acts related with personal or industrial accidents and to design improvement or prevention strategies. This technique at natural contexts requires as much rigor in the design of the behavioral inventory or checklist as in its application. Methodological problems, as well as advantages and disadvantages to use this technique at organizational contexts are analyzed. How the results from to apply observations affect organizational psychosocial interventions, are discussed too. Observations could use with other techniques from another approaches. Probably technique utility depends somehow to the organizational safety culture, culture that itself procedure promote to prevent and to control the occurrence of future accidents in the companies.

Key words author:

Safe behaviors, unsafe behaviors, unsafe acts, behavior observation, prevention, safety.

Key words plus:

Accidents, unsafe acts, behavior observation, organizational psychology, behavioral assessment. 


\section{Introducción}

La observación y registro de conductas inseguras y seguras de los trabajadores en los ambientes de trabajo, ha sido un procedimiento empleado en los últimos años en muchas empresas, conocido con el nombre de "Seguridad basada en el Comportamiento", como una estrategia fundamental para la detección y medición de los actos inseguros que incrementan la probabilidad de ocurrencia de accidentes personales o industriales en dichos ambientes, así como para diseñar estrategias de intervención para su prevención y control (Behavioral Science Technology, 1994; Cox \& Jones, 2006; Fleming \& Lardner, 2002 citados por Anderson, 2005; Paul \& Maiti, 2007; Petróleos de Venezuela, 2002; Step Change in Safety, 2000; Wirth \& Sigurdsson, 2008). No obstante, el uso de esta técnica en ambientes naturales requiere mucha rigurosidad, tanto en el diseño de la observación y elaboración de registros o listas de verificación de conductas como en la aplicación de la misma por observadores adecuadamente entrenados.

Dentro de las organizaciones, estos observadores suelen ser supervisores o trabajadores parcialmente entrenados, que deben observar y registrar las conductas inseguras y seguras de otros trabajadores que conforman el grupo de trabajo objeto de observación, mientras realizan sus actividades de trabajo regulares y cotidianas, para luego proveerles realimentación sobre las mismas. Aun cuando se garantice el anonimato de los registros y se incorpore el acuerdo inter-observadores para garantizar la confiabilidad de las mediciones realizadas, se corre un gran riesgo de que los observadores y observados introduzcan su sesgo personal, ya que también son trabajadores o supervisores, estos últimos con autoridad para tomar acciones sobre su personal lo que puede influir en el comportamiento de sus supervisados, debido a las expectativas de consecuencias laborales (positivas o negativas) que en los mismos puedan generarse.

Recientemente, Wirth y Sigurdsson (2008) realizaron un análisis de las diferentes áreas de atención de los programas o procesos de seguridad basada en el comportamiento que requerirían de investigación científica. Específicamente, respecto a la observación y medición, estos autores consideran que es importante conocer si los registros y observaciones conductuales son la mejor forma de medir la ejecución segura o hay métodos alternativos; qué número de conductas a observar es el óptimo para ser incluido en un registro o lista de verificación a emplear durante la observación y si este número afecta o no la precisión de la medición; si la frecuencia con la que se realizan las observaciones afecta la precisión o confiabilidad de la medición y cuál sería la frecuencia mínima de observaciones efectiva; si los observadores pueden o no concentrarse más en verificar las conductas seguras (efecto del observador) y qué condiciones o variables pueden influirlos. En lo que se refiere al empleo de la realimentación ofeedback, se preguntan si esta siempre es necesaria para el cambio conductual y bajo qué condiciones sería fundamental aplicarla; si su frecuencia, inmediatez o duración impacta o no la efectividad de la intervención; si el modo o tipo de realimentación (p. ej., verbal o escrita, tangible o intangible, específica o general, pública o privada) es importante o no; si es importante o no quién la provee (p. ej., los compañeros de trabajo, supervisores o gerentes); si debería ser individualizada para cada trabajador y bajo qué condiciones sería efectivo proporcionarla en grupo.

El propósito de este artículo es analizar algunos de los problemas metodológicos, así como reflexionar sobre las ventajas y desventajas del uso de la técnica de observación para la identificación, medición y registro de las conductas de seguridad o inseguridad de los trabajadores en ambientes organizacionales, donde también por mucho tiempo ha predominado un paradigma cultural que podríamos denominar "la búsqueda de culpables", o como refieren Cox y Jones (2006) una tendencia en las organizaciones caracterizada por culpar o criticar a los individuos o grupos ante la ocurrencia de accidentes de trabajo, atribuidos en gran parte a dichas conductas inseguras. Así mismo, es de interés mencionar las implicaciones que tiene la poca rigurosidad en la aplicación de la técnica, para la puesta en práctica de intervenciones psicosociales en una organización. 


\section{Antecedentes}

La aplicación de las ciencias del comportamiento, en especial de la Psicología y particularmente del enfoque conductual de la misma, así como de las técnicas de modificación de conducta, al estudio e intervención del comportamiento humano organizacional relacionado con la prevención de accidentes, se remonta a los trabajos de Komaki, Barwick y Scott (1978), en los que estas técnicas se introducen de manera sistemática, para mejorar los resultados de las prácticas de seguridad industrial en los ambientes laborales. Estos representan una de las aplicaciones de los principios del aprendizaje humano demostrados por B. F. Skinner a partir de 1940 y del Análisis Conductual Aplicado desarrollado posteriormente, los cuales son fundamentos del enfoque conductual en Psicología. En los mismos, se contempla la definición de conductas relacionadas con la seguridad (seguras y riesgosas) en términos observables, el uso de la observación sistemática como técnica para su medición y registro en ambientes laborales y la administración de la realimentación (feedback) verbal a los trabajadores, como mecanismo para promover su modificación, aunado al manejo instrumental de las consecuencias de la conducta en dichos ambientes. Es decir, se parte de que las conductas seguras o inseguras se aprenden y se mantienen por las consecuencias que tienen en el ambiente; por tanto, si se cambian las consecuencias pueden modificarse las conductas.

Posteriormente, autores tales como Krause, Hidley y Hodson (1990), McSween (1995) y Geller (1996, 2000, 2001) también han aplicado este y otros enfoques y métodos de las ciencias del comportamiento, a través de procedimientos mejores, adaptados a las realidades laborales de las grandes industrias (conocidos en inglés como The Behavioral Accident Prevention Process, The BehaviorBases Safety Process, The Value-Based Safety Process, Behavior-Based Safety, entre otros), los cuales han sido incorporados por empresas líderes en el ámbito de la industrias petrolera, manufacturera, electrónica, metalmecánica, del papel, química, entre otras, con resultados exitosos e impactos favorables en la reducción de la accidentalidad (Behavioral Science Technology, 1994; Step Change in Safety, 2000).

Estos procedimientos contemplan el empleo del método y la técnica de la observación como medio de identificación y medición de las conductas seguras y riesgosas en el ambiente de trabajo, con miras posteriormente a la búsqueda de la reducción de las segundas y reforzamiento e incremento de las primeras, como parte de las acciones para la prevención de accidentes. La aplicación de estos procedimientos permite conocer los actos inseguros que podrían estar asociados a la ocurrencia de accidentes en un determinado puesto de trabajo, antes de que estos ocurran; tradicionalmente, en las organizaciones los actos inseguros se han inferido de la investigación y análisis que se realiza luego de ocurrido un evento no deseado. También contribuye a que las conductas inseguras se anticipen, observen, registren y se retroalimente verbalmente a los trabajadores en el sitio de trabajo, durante la ejecución de las tareas en el ambiente laboral, para hacerles seguimiento antes de que ocurra un evento inesperado. Además, conlleva a que se analicen los eventos o factores antecedentes que propician conductas seguras y de riesgo en el trabajo, así como los resultantes que las refuerzan, para introducir correctivos en el ambiente de trabajo o en aspectos como la supervisión de los trabajadores (Krause et al., 1990).

\section{Observación de la conducta en ambientes naturales}

Antes de analizar metodológicamente los procedimientos seguidos por algunas organizaciones, para la observación de conductas inseguras en el trabajo, conviene realizar una revisión de los aspectos que distinguen a la observación como método y técnica de abordaje científico de un objeto de estudio, así como las dificultades propias de esta estrategia de evaluación conductual. Tal y como lo expresan Anguera (1981a), Anguera (1991) y Blanco, Losada y Anguera (2001) el método de la observación implica una serie de características básicas que pueden resumirse así: 
a. No hay distorsión o preparación de la conducta a observar, lo que implica que debe transcurrir en forma natural y como aparece a los ojos del observador.

b. Debe establecerse un muestreo previo: qué se va a observar y a quién o quiénes se van a observar.

c. No existe manipulación de la conducta observada, es decir no se introduce ninguna variable experimental.

d. Se deja constancia de la conducta observada, a través de un registro adecuado que permite generar un análisis cuantitativo y cualitativo.

Así mismo, estos autores señalan que existen algunas dificultades metodológicas conocidas y que se deben tener presentes cuando se recurre a este método, tanto en ambientes naturales como de laboratorio. Estas se concentran en el observador, el observado, la validez y confiabilidad en el uso del método. Mencionan como sigue:

a. Las provenientes del propio observador, tales como el sesgo inevitable en su percepción y en la interpretación de lo percibido; el conocimiento previo de la situación y de la conducta observada, que introduce un filtro selectivo de lo que se observa; la falta de formación o preparación para observar que provoca diferencias en los registros de conducta y los cambios que ocurren en el observador durante las diferentes sesiones de observación. Estos errores podrían subsanarse con una adecuada formación del observador en las características de la técnica y la suficiente preparación a través de ensayos o practica previa.

b. Las relacionadas con la persona observada, tales como el sesgo que introduce en su comportamiento por su reactividad ante la presencia del observador, lo cual varía dependiendo del grado de intromisión del procedimiento, y la reactividad recíproca que se genera cuando la conducta del observado afecta de alguna manera la percepción y conducta del observador. Este sesgo pudiera disminuir con la habituación de los observados ante el observador o con la renovación de los observadores. c. Las relacionadas con la validez de la observación, que se refieren a la necesidad de que existan códigos comunes de observación y criterios observacionales para el registro, lo que implica la definición operacional de las conductas a observar, en términos descriptivos que faciliten su identificación y diferenciación en la persona observada. Así como la aplicación de procedimientos psicométricos para establecer la validez de contenido, concurrente y de constructo.

d. Las vinculadas a la confiabilidad de la observación, que indican la necesidad de contar con observadores simultáneos y similitud entre los registros de los mismos (acuerdo entre observadores), donde debe existir una proporción aceptada de ítems de observación en los cuales estos coinciden.

De manera específica, en cuanto a la observación de conductas en ambientes naturales, Anguera (1981b) y Anguera (1991) también señala otros aspectos de suma importancia, que se podrían vincular directamente con la observación de conductas inseguras y seguras en ambientes laborales u organizacionales:

a. La conducta en el trabajo está fuertemente condicionada por el entorno, lo que puede influir en la espontaneidad de las conductas de las personas en el momento de realizar la observación, aunque el grado de sistematización y rigurosidad de la observación, así como del registro, sea alto.

b. Deberían emplearse procedimientos de registro que no requieran interferencia (p. ej. videos), sin intromisión o indirectos.

c. Puede perderse información (conductas) que no se haya considerado en los ítems del sistema de categorías de observación previamente diseñado; no necesariamente la muestra de datos recolectada llega a ser la más representativa de la conducta o área de conducta a observar y tampoco el acuerdo entre observadores, de aplicarse, garantiza que el observador registró realmente la conducta que emitió el trabajador 
(es decir la alta confiabilidad no garantiza la alta validez de la observación).

d. La reactividad originada en el proceso de observación cuando se conoce o infiere (supone) el objetivo y hasta las posibles consecuencias de la observación.

e. El sesgo potencial del observador, promovido por diferentes aspectos tales como: la expectativa de resultados acerca de la observación, la realización de un posible feedback evaluativo, el conocimiento en sitio del grado de acuerdo existente entre los observadores, el efecto de su sola presencia o instrucción dada al observado en la ecología o naturalidad de la situación a observar, la familiaridad que se va adquiriendo con el observado que puede afectar la confiabilidad y cualquier evento externo o interno que afecte la supuesta estabilidad de las observaciones realizadas.

\section{Problemas metodológicos en su aplicación}

Con base en las características de la observación, dificultades metodológicas conocidas y particularidades de su aplicación en ambientes naturales mencionadas con anterioridad, se analiza el procedimiento de observación de conductas seguras e inseguras en el trabajo, que han venido aplicando en los últimos diez años algunas organizaciones en el mundo.

Este procedimiento en la práctica, más o menos uniformemente entre un autor y otro (Krause et al., 1990; McSween, 1995; Geller, 1996, 2000, 2001) o entre una empresa consultora y otra (Behavioral Science Technology, Inc.; Quality Safety Edge; Safety Performance Solutions, Inc.), tal y como lo muestran Wirth y Sigurdsson (2008, p. 591) en un mapa genérico del mismo, requiere la participación de todos los niveles organizacionales e implica el diseño de un proceso para la observación de conductas seguras e inseguras, que son desempeñadas por los trabajadores regulares en sus ambientes naturales y cotidianos de trabajo (talleres, plantas, oficinas, laboratorios, instalaciones externas, entre otros). Cada observación puede ser realizada a un solo tra- bajador o a un conjunto de trabajadores (p. ej. una cuadrilla o equipo de trabajo). Considera la realización previa de un análisis funcional de la conducta de seguridad o inseguridad de estos trabajadores, que debe realizar un equipo de diseño constituido por conocedores del puesto y del procedimiento, el cual conlleva a:

- La identificación de las conductas críticas por incrementar o reducir.

- La elaboración de registros de observación (por lo general listas de verificación o chequeo) donde se agrupan por categorías estas conductas críticas identificadas y definidas operacionalmente.

- El entrenamiento de los observadores para el uso adecuado del registro diseñado y la aplicación del procedimiento de realimentación de las conductas seguras e inseguras observadas.

- La observación de las conductas críticas en el trabajador o trabajadores que desempeñan un puesto en un ambiente de trabajo seleccionado, haciendo uso del registro previamente diseñado.

- El establecimiento de una medición de línea base en función de la frecuencia de aparición de dichas conductas (lo que aproximadamente puede representar cuatro semanas de levantamiento de datos, según Krause \& Hidley, 1990).

- La realización de intervenciones de la conducta en la dirección deseada (sea en la modificación de los factores antecedentes o en las consecuencias de la conducta).

- La aplicación por parte del observador de la realimentación verbal modificadora (feedback verbal modificador), cada vez que observe conductas de riesgo o inseguras de parte de los trabajadores.

- La prueba del impacto de las intervenciones con la realización de observaciones y registros continuos, durante el proceso de cambio de conductas de riesgo o inseguridad a conductas de seguridad.

- La continuidad de las observaciones como un proceso de mejoramiento en el tiempo de la conducta y cultura de seguridad de la organización. 
Varios aspectos en el procedimiento se pueden catalogar como problemas metodológicos, así:

1. La realimentación verbal de la conducta observada durante el proceso de observación, despierta duda sobre la rigurosidad que pueda tener la aplicación del método y técnica de la observación en este tipo de procedimiento, puesto que significa la introducción de una variable, si no experimental, interviniente en la naturalidad de la conducta (aunque la intención sea proporcionar realimentación ante conductas de riesgo).

2. El hecho de que los observadores que registran las conductas para el establecimiento de la línea base, son supervisores o trabajadores y no observadores neutrales no sesgados, pudiera estar distorsionando el resultado de las observaciones, haciendo que este se aleje de la realidad. Al respecto, Krause et al. (1990) consideran que el observador ideal es la persona que tiene alta credibilidad entre sus pares (es decir un compañero de trabajo), que conozca el trabajo que se va a observar (que podría ser el mejor trabajador o el supervisor inmediato) y que posea adecuadas destrezas interpersonales y de comunicación. El asunto está en que la sola presencia de trabajadores o supervisores, conocidos por los trabajadores observados, durante el proceso de observación, o específicamente como observadores que registran conductas, altera de alguna manera la conducta cotidiana de los trabajadores que se pretende registrar. Más, si las observaciones son comunicadas con antelación a su realización en aras de lograr un ambiente de confianza, como en algún caso se sugiere (Geller, 2001).

3. Estos dos aspectos mencionados parecieran indicar que la rigurosidad en la medición queda en un segundo plano. El hecho de dialogar con el trabajador y comunicarle la importancia del proceso de observaciones, realimentarle verbalmente lo inadecuado en caso de que se observe, indicándole lo adecuado en el momento de la observación, afecta negativamente el proceso de observaciones. Sería difícil detectar lo que general y regularmente están haciendo en forma segura o insegura los trabajadores al realizar sus tareas.

4. Esta falta o poca rigurosidad en la observación, conllevaría a no tener un resultado cercano a la realidad cotidiana de trabajo, por lo que el tipo e intensidad de las intervenciones que se planifiquen no serían las realmente requeridas; por tanto, se estaría haciendo poco para que el proceso de observaciones tenga el resultado esperado, que es el control de la conducta para la disminución de la ocurrencia de eventos no deseados en el trabajo, como son los accidentes.

5. Pareciera que por la naturaleza de la conducta (una conducta insegura puede incrementar el riesgo de un evento no deseado, tenemos que corregirla o evitarla en el momento que la observamos, para que esta probabilidad no incremente o que el evento ocurra), se hace difícil el registro de su frecuencia en el tiempo en ambientes naturales. También es probable que la conducta insegura en el observado se repita a pesar de las indicaciones o correcciones dadas por el observador, lo que lleva a cuestionar o reflexionar en cuanto a la potencia del feedback verbal, para la modificación de la conducta en el tiempo.

6. En cuanto a los registros de observación utilizados, estos solo sirven para verificar conductas. No se indican formatos de registro de antecedentes críticos que facilitan conductas inseguras y de las consecuencias que los mantienen (factores del ambiente físico y social laboral, o del individuo). Estos últimos permitirían conocer, ante una conducta insegura observada, si podemos disminuir su frecuencia pero no extinguirla, ya que sigue siendo reforzada y facilitada por el ambiente que rodea el puesto de trabajo. Esto es, se le puede decir al trabajador lo que hace en forma insegura y cómo lo debe hacer bien o en forma segura, pero si no se registran y controlan los antecedentes y consecuencias de esa conducta en el ambiente laboral, la misma probablemente se repetirá.

En síntesis, aun cuando los procedimientos de seguridad basada en el comportamiento recurren a 
la observación de la conducta en el medio natural, es decir, en los puestos de trabajo donde la conducta de seguridad o inseguridad se desempeña, corren el riesgo de que la conducta que se observe esté distorsionada por el efecto que introduce el observador al conversar con el trabajador, comunicarle cuál es el objetivo de su presencia y, más aún, al proveerle el feedback verbal modificador, cuando haya observado conductas inseguras en su desempeño. Como se apuntó anteriormente, esto podría intervenir como una variable que se introduce en el contexto de la observación y que puede afectar la conducta del trabajador, lo cual no es propio en el empleo de la observación como método o técnica de registro. Es probable que luego de la observación y el feedback proporcionado, el trabajador comience a trabajar como nunca lo ha hecho, cumpliendo las normas de seguridad que conoce pero que por hábito, ahorro de tiempo o esfuerzo en su comportamiento cotidiano, no lo hace. Es el caso de los trabajadores que se ponen el casco o los guantes cuando ven o son vistos por el supervisor, pero después se los quitan argumentando calor o incomodidad. La pregunta es: iel procedimiento logra registrar e intervenir el comportamiento cotidiano del trabajador?

Este aspecto de acercamiento a la cotidianidad, también cobra importancia en las observaciones que se realizan para registrar la línea base de la conducta; no es extraño que en algunas mediciones se puedan reflejar contradicciones entre los resultados de las mediciones para establecer la línea base y los subsecuentes registros de observación, donde una conducta insegura no se observa con muy alta frecuencia en la línea base y luego en observaciones posteriores llega a incrementar. O que en puestos de trabajo donde han ocurrido accidentes que están relacionados con una conducta insegura en particular, como por ejemplo el uso de una herramienta de trabajo inadecuada o la omisión en el uso del equipo de protección personal, esta conducta no sea registrada en las observaciones para la línea base. Que el observador registre el comportamiento seguro o inseguro más cercano a lo cotidiano en el trabajo, es el reto de estos procedimientos.

Otros problemas metodológicos que se detectan se relacionan con:
1. La preparación del observador en cuanto a las tareas del puesto de trabajo a observar; este debe ser conocedor del mismo, y particularmente de los momentos críticos donde puede haber la tendencia al error o a la conducta insegura. Este mismo conocimiento introduce un sesgo en la selección de lo observado, el filtro selectivo del que habla Anguera (1981 citado en Fernández \& Carrobles, 1981), que dirige la observación hacia lo que sabe o conoce el observador. Esto es muy importante, si se toma en consideración que el trabajador al realizar todos los días su trabajo, acumula suficiente experiencia en la ejecución del puesto, lo que desarrolla en él un nivel de conocimiento sobre el mismo que tal vez no tenga el observador, sobre todo si este es un supervisor quien hace ya un tiempo dejó de interactuar cotidianamente con la ejecución de los puestos de trabajo. Cada puesto de trabajo, actividad y tarea tienen su particularidad y su especificidad, y el que lo desempeña todos los días es el que puede informar sobre esos momentos críticos de su trabajo, que tengan que ver con la seguridad laboral.

2. El riesgo es que quienes observan y registran conductas no tengan la suficiente preparación sobre el puesto de trabajo como para observarlo adecuadamente, lo que afectaría la calidad de la información registrada. Por tanto, en un entrenamiento de observadores, debería estar presente un trabajador de alto desempeño y experiencia en el puesto, para poder equilibrar la información teórica y práctica que se le proporciona al observador. El conocimiento de las variables que afectan la técnica de observación también es fundamental para aplicarla eficientemente. Por lo general, en los procedimientos reseñados esto se descuida y el entrenamiento del observador se limita al conocimiento y manejo de la hoja de registro o lista de verificación de conductas a observar, así como en la forma de proveer la realimentación verbal al trabajador o trabajadores que observen.

3. La metodología propuesta por los autores mencionados (Geller, 1996, 2000, 2001; Krause et al., 1990; McSween, 1995), indica la necesidad 
de realizar un análisis previo de las tareas del puesto de trabajo, que permita identificar las conductas críticas que formarán parte de la hoja de observación o lista de verificación, lo que pareciera indicar también la necesidad de registros ad hoc o específicos para cada puesto de trabajo. No obstante, se tiende a emplear como registro una hoja común que permite estandarizar los ítems de observación para todos los puestos de trabajo, sacrificando de esta manera las particularidades que en prácticas de trabajo seguro tiene cada puesto, las cuales orientan no solo la conducta propia del trabajador, sino los eventos antecedentes y consecuencias de una conducta segura o insegura. Algunos incorporan el registro de algunas prácticas de trabajo seguro, que se han encontrado relacionadas con la accidentalidad en el desempeño del puesto (McSween, 1995).

4. El procedimiento establece un muestreo previo de qué se va a observar y en quiénes y dónde, es decir un diseño de la observación. En la práctica se corre el riesgo de que el muestreo no sea representativo de la realidad, debido a los cambios diarios y mensuales propios del mundo laboral (guardias, turnos, vacaciones, permisos, etc.). Es posible que se observe a los mejores y seguros trabajadores o a los inseguros, si estos llegan a coincidir en el momento de la observación, lo que no daría una aproximación a lo que en realidad ocurre en un puesto de trabajo o en un ambiente laboral. Habría que ampliar suficientemente el período de observaciones, para ejercer algún control.

5. El conocimiento previo por parte de algunos trabajadores de que las observaciones se van a realizar, introduce un sesgo selectivo en los responsables de los grupos de trabajo; es decir, ya no sería solamente la reactividad del observado ante la presencia del observador, la cual puede neutralizarse con el anonimato de los registros (lo cual sí está contemplado en el procedimiento), sino la "reactividad defensiva", por llamar de alguna manera al proceder del que cree perder autoridad o poder en la organización, si se observan y registran conductas inseguras en los trabajadores bajo su responsabilidad.

\section{Ventajas y desventajas del procedimiento}

El análisis del procedimiento seguridad basada en el comportamiento también ha permitido reflexionar sobre algunas de sus ventajas y desventajas:

\section{Ventajas:}

- Permite la recolección de información sobre conductas inseguras específicas que podrían modificarse mediante aprendizaje de las conductas seguras.

- La estadística acumulada, producto del proceso de observaciones, contribuye a orientar acciones de intervención y seguimiento de la conducta de los trabajadores, para mejorar la seguridad y salud en el trabajo.

- Podría estimular el desempeño adecuado del rol de supervisor en cuanto a la seguridad en el trabajo, así como ampliar su alcance.

- Ayuda a los trabajadores poli-accidentados a evitar futuras lesiones.

- Contribuye a reconocer conductas inadecuadas en los trabajadores mientras realizan sus tareas, y promover el mantenimiento de la salud y seguridad en el trabajo.

\section{Desventajas:}

- Todos los problemas metodológicos mencionados con anterioridad.

- Resultados concretos pueden verse en un largo plazo, cuando el procedimiento logra insertarse en la cultura de seguridad de la organización.

- Requiere el entrenamiento y ocupación de muchos trabajadores en la tarea de observación de conductas y procesamiento de registros, lo que ocasiona en algunos casos aumento de la carga de trabajo dentro de los grupos en la organización.

- El empleo de hojas de registro o listas de verificación genéricas aplicables a la observación de cualquier puesto de trabajo.

- Se concentra en el aspecto molecular de la conducta, descuidando el efecto del entorno. 
- El cambio conductual en seguridad y salud laboral no solo se estimula desde afuera (antecedentes y consecuencias de la conducta en el ambiente de trabajo), sino también interviniendo factores personales relacionados con la conducta de riesgo, como por ejemplo la percepción individual del riesgo (sobreestimación o subestimación de los riesgos al desempeñar un puesto de trabajo).

- El sesgo que introduce la deseabilidad social: cuando una persona es observada se comportará como se espera que lo haga.

- Los roles organizacionales de quienes observan o son observados dentro de la estructura de autoridad de la organización, podrían afectar el resultado de la observación conductual.

- Al no generar resultados a corto plazo, dentro de los grupos de trabajo el procedimiento se percibe más como una carga que como una solución.

\section{Conclusiones y recomendaciones}

Hasta aquí, se ha visto que el procedimiento tiene un alto grado de intromisión por parte del observador, aun cuando se plantea que la continuidad de las tareas no se debe alterar mientras se realiza la observación. El efecto inevitable de la presencia del observador sobre el observado y de la conducta del observado sobre el observador, podría influir en el resultado de la observación, pero este efecto también podría reducirse con la "familiaridad" que se establezca entre ellos, lo que lleva a pensar en la posibilidad de complementar este procedimiento de Seguridad basada en el Comportamiento con métodos de observación propuestos en otros paradigmas distintos al positivista y conductual, tales como la observación participante de la Etnografía, que es mucho más invasiva pero mucho más "familiar" en la relación sujeto-objeto. Todo con miras a lograr el acercamiento más real posible a la complejidad del fenómeno de las conductas inseguras en los ambientes organizacionales, lo cual podría ocurrir si se eleva la espontaneidad de la conducta a observar.

Dentro del paradigma conductual y psicométrico, llevar a cabo un acuerdo entre-observadores de manera adecuada, con suficientes observadores, sesiones de observación y registros simultáneos de un mismo trabajador o grupo de trabajadores observados, y hasta la incorporación de medios que no impliquen interferencia, elevaría el nivel de confiabilidad de la observación realizada al no permitir la familiaridad y controlar la reactividad observadorobservado, pero no necesariamente incrementaría la espontaneidad de la conducta. De igual manera, no garantiza que la realidad no sea distorsionada por los intereses de sus participantes, y dentro de las organizaciones es frecuente que variables como el poder y el miedo tengan más influencia que la prevención y la seguridad laboral.

Participar en un grupo de trabajo por mucho tiempo como observador, pudiera incrementar la posibilidad de captar comportamientos espontáneos de los trabajadores, así como descifrar el efecto ineludible del entorno en los mismos. Otro tipo de técnicas, como por ejemplo la del "foto-safari" a través del uso de la fotografía de tecnología digital (Danish Technological Institute, s.f.; Oncins \& Murillo, s.f.), entre cuyos objetivos se encuentra la identificación de actos inseguros o situaciones peligrosas, derivadas fundamentalmente del comportamiento humano, pudiera contribuir a detectar esos comportamientos espontáneos.

La seguridad basada en el comportamiento en las organizaciones ha pretendido ser un procedimiento que promueve a largo plazo cambios importantes en la cultura de seguridad de los trabajadores de todos los niveles y jerarquías; pero su ejecución, resultados y utilidad se ven fuertemente influidos por variables del entorno laboral y por la cultura predominante en la organización. Es promover una nueva manera de hacer las cosas sobre la forma vieja de hacerla; el solo registro de las conductas y el mantenimiento en el tiempo de un proceso de observaciones generador de información, que permita tomar medidas preventivas con el comportamiento de los trabajadores, no es suficiente si el proceso de cambio no alcanza de manera simultánea a los factores que anteceden y siguen a la ejecución de conductas de riesgo o inseguras en el trabajo.

Si el proceso de observaciones no comienza a ser parte del rol y función tradicional y cotidiana de un supervisor, así como si los trabajadores no incorpo- 
ran en su repertorio de conductas en el trabajo la de observar para proteger a sus compañeros de trabajo, las posibilidades del cambio cultural son escasas. Es posible que un criterio pragmático para la aplicación del método de observación, haya prevalecido en los autores que propusieron este procedimiento; no obstante, no deben pasarse por alto los aspectos discutidos hasta aquí, para contribuir a optimizarlo o desarrollar otro tipo de estrategias de abordaje de la problemática de la seguridad y salud en el trabajo.

Puede concluirse que la utilidad de la técnica en ambientes naturales responde de alguna manera a la cultura de seguridad existente en la organización, cultura que el mismo procedimiento persigue promover, para prevenir y controlar la ocurrencia de futuros accidentes en las empresas. Así mismo, depende de la corrección de los sesgos e imperfecciones en el diseño y ejecución de las observaciones, en puestos de trabajo disímiles.

La observación de conductas seguras e inseguras en el trabajo puede emplearse como una técnica complementaria a otro tipo de mediciones de variables del comportamiento asociadas a la seguridad en el trabajo, y no como "el método" para la prevención de los factores psicosociales y conductas humanas asociadas a la ocurrencia de accidentes o enfermedades ocupacionales.

\section{Referencias}

Anderson, M. (2005). Behavioral safety and major accident hazard: Magic bullet or shot in the dark? Process Safety and Environmental Protection, 83(B2), 109-116.

Anguera, M. T. (1981a). La observación (I): problemas metodológicos. En R. Fernández Ballesteros \& J. A. Carrobles (Eds.), Evaluación Conductual. Metodología y aplicaciones (pp. 292-333). Madrid: Pirámide.

Anguera, M. T. (1981b). La observación (II): situaciones naturales y de laboratorio. En R. Fernández Ballesteros \& J. A. Carrobles (Eds.), Evaluación Conductual. Metodología y aplicaciones (pp. 33363). Madrid: Pirámide.
Anguera, M. T. (1991). Evaluación del comportamiento en contextos naturales. Revista de Psiquiatría de la Facultad de Medicina de Barcelona, 18(6), 277-287.

Blanco, A., Losada, J. I. \& Anguera, M. T. (2001). Diseños observacionales: cuestión clave en el proceso de la metodología observacional. Metodología de las Ciencias del Comportamiento, 3(2), 135-160.

Behavioral Science Technology. (1994). An ongoing study of the behavioral accident prevention process (Initial Report). Austin, TX: Autor.

Cox, S. \& Jones, B. (2006). Behavioural safety and accident prevention: Short-term 'Fad' or sustainable 'Fix'? Process Safety and Environmental Protection, 84(B3), 164-170.

Danish Technological Institute. (s.f.). Photo Safari: A learning platform for organisational benchmarking. Recuperado el 01 de mayo de 2009, de http://www. innoflex.ora.uk/

International Association of Oil and Gas Producers. (2001). Human Factors: A way to improve performance. London: Autor.

Komaki, J., Barwick, K. D. \& Scott, L. R. (1978). A behavioral approach to occupational safety: Pinpoiting and reinforcing safety performance in a food manufacturing plant. Journal of Applied Psychology, 63(4), 434-445.

Krause, T. R., Hidley, J. H. \& Hodson, S. J. (1990). The behavior-based safety process. New York: Van Nostrand Reinhold.

Geller, E. S. (1996). The psychology of safety: How to improve behaviors and attitudes on the job. Boca Raton, FL: CRC Press.

Geller, E. S. (2000, junio). How to sustain involvement in occupational safety: From research-based theory to real-word practice. En Memorias de la Conferencia Internacional de la American Society Safety Engeniers (ASSE), Orlando, Florida, EE.UU.

Geller, E. S. (2001). The psychology of safety handbook. Boca Raton, FL: CRC Press.

McSween, T. E. (1995). The value-based safety process. Improving your safety culture with a behavioral approach. New York: Van Nostrand Reinhold.

Oncins de F., M. \& Murillo, B. (s.f.). Foto-safari: una herramienta de observación en el trabajo. Recuperado el 01 de mayo de 2009, de http://www.mtas.es/ insht/ntp.709 
Paul, P. S. \& Maiti, J. (2007). The role of behavioral factor on safety management in underground mines. Safety Science, 45, 449-471.

Petróleos de Venezuela, S.A. (2002). Norma SI-S-10. Seguridad basada en Comportamiento (SBC) (Norma interna de Seguridad Industrial). Caracas: Autor.

Step Change in Safety. (2000). Changing Minds. A practical guide for behavioural change in the UK oil and gas industry. Aberdeen, UK: Autor. Disponible en http://www.stepchangeinsafety.net/knowledgecentre/publications/publication.cfm/publicationid/16 Wirth, O. \& Sigurdsson, S. O. (2008). When workplace safety depends on behavior change: Topics for behavioral safety research. Journal of Safety Research, 39, 589-598. 
